\title{
Experimental study of Reflectance Spectrophotometric Analysis of the Dog's Dural Sac-in the Open Decompressed State and after the Application of External Dural Pressure.*
}

\author{
N. Tajima, MD, K. Kawano, MD, K. Matsumoto, MD, K. Tashiro, MD, \\ K. Saisho, MD \\ Department of Orthopaedic Surgery, Miyazaki Medical College, 5200 Kihara, \\ Kiyotake, Miyazaki 889-16, fapan.
}

\section{Summary}

Haemoperfusion and oxygen saturation were studied by tissue reflection spectra on the dural sac of dogs, Experimentally, decompression laminectomy and external pressure on the dural sac were carried out. Haemoperfusion and oxygen saturation were investigated in the open state, and after an external pressure was applied. After the application of external pressure, paraplegia occurred.

The tissue Spectrum Analyser 'TS-200' (Sumitomo Electric Industries Co. Ltd, fapan) was used for the measurement of haemoperfusion and oxygen saturation. With this apparatus, an optical fibre probe is applied to irradiate tissue with white light, and the absorption spectrum of the reflected light from tissue is analysed.

It has been confirmed that oxygen saturation was not changed in either state, however, haemoperfusion was decreased when external pressure was applied.

Key words: Spinal cord compression; Spectrophotometry; Haemodynamics.

To elucidate circulatory changes in the dural sac in paraplegia, we studied experimental circulatory changes in the dura mater as indicated by the haemoglobin level in the blood by using a reflected spectrum that allows quantitative determination of the oxygen metabolism in tissues.

The tissue reflectance spectrum technique that was developed by Chance et al. in 1951 to determine oxidation and reduction of dyes in tissue employs a two wavelength spectrophotometer. Thereafter, Sato et al. developed an instrument for organ reflectance spectrum analysis, which is now used to observe mucosal haemodynamics in combination with an endoscope.

* Presented at 27th Annual Scientific Meeting of IMSOP, Perth, WA, Australia, 10 May 1988. 


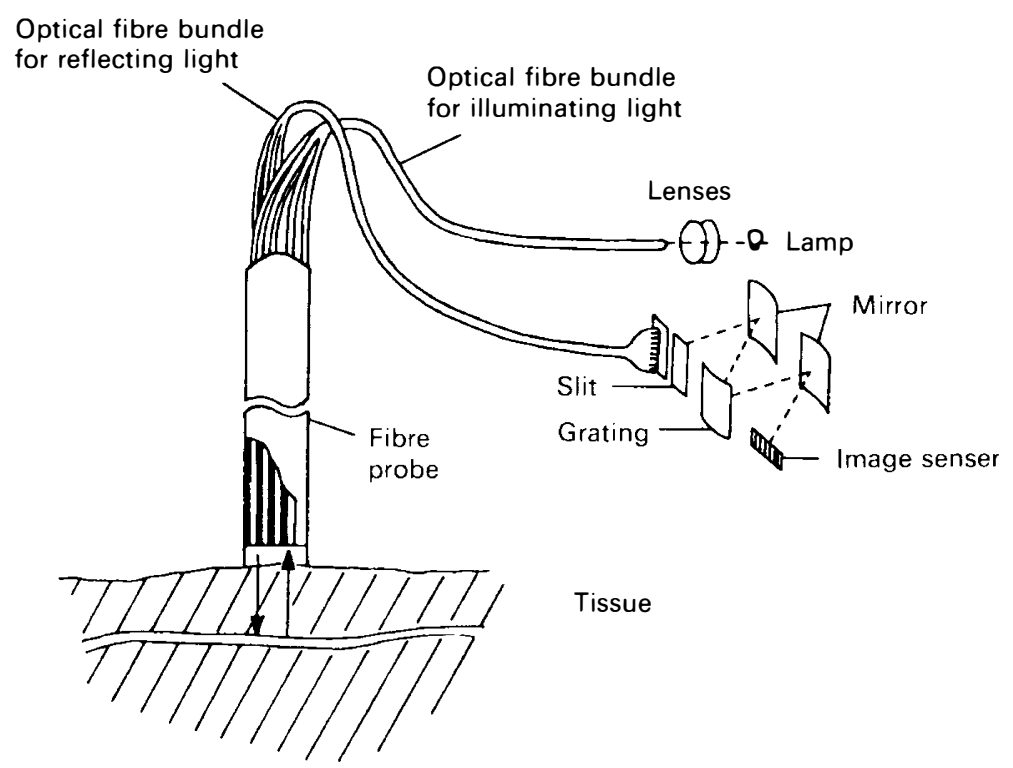

Figure 1 Spectrometry system. (Tissue Spectrum Analyzer 'TS-200' Sumitomo Electric Industries Co. Ltd.).

\section{Materials and methods}

\section{Subjects}

Measurements were made in the dural sac of 5 dogs in the open decompression state due after laminectomy, and after extenral dural pressure was applied. The experimental dógs' weights were 15-18 kg. Under general anaesthesia, laminectomy of the lumbar spine was carried out.

In the present experiment, a fibre holder with a spring exerted a fixed pressure on the dura mater, and a constant was determined under compression of $400 \mathrm{~g} / 20 \mathrm{~mm}$ (20 g/unit). An external dural pressure of $600 \mathrm{gm}$ was applied.

\section{Reflectance spectrophotometric analysis}

A tissue spectrometer 'TS-200' (Sumitomo Electric Industries Co. Ltd, Japan) was used. A fibre probe was attached to the surface of the dura mater, enabling the level of haemoglobin and the extent of oxygen saturation to be determined non-invasively and rapidly (Fig. 1).

The probe containing a fibre-optic bundle was attached to tissues to irradiate white light and analyse the absorption spectrum of tissues according to the reflected light. This computer-assisted method provided the time course and quantitatively based imaging of changes in pigments in tissues in the form of a continous spectrum. The difference in logarithm between the reflected spectrum for the standard white plate and that for the subject was calculated as the absorption spectrum.

Accordingly the extent of oxygen saturation $\left(\mathrm{SO}_{2}\right)$ of haemoglobin changes from oxidation to reduction and from reduction to oxidation, and shows changes in the spectrum at $569-586 \mathrm{~nm}$. 
Table Measurements of oxygen saturation and haemoglobin concentration in the dura sac in the open decompressed state and af ter external dural pressure was applied.

\begin{tabular}{lccc}
\hline & & $\begin{array}{c}\text { Open } \\
\text { decompressed state }\end{array}$ & $\begin{array}{c}\text { After external } \\
\text { pressure was applied }\end{array}$ \\
\hline Oxygen saturation $\left(\mathrm{ISO}_{2}\right)$ & No.1 & $39.9 \pm 2.0$ & $44.6 \pm 1.4$ \\
& No. 2 & $44.0 \pm 1.5$ & $43.4 \pm 3.6$ \\
& No. 3 & $36.8 \pm 1.4$ & $33.8 \pm 2.7$ \\
Haemoglobin concentration $(\mathrm{IHb})$ & No. 4 & $34.3 \pm 1.3$ & $42.0 \pm 9.8$ \\
& No. 5 & $27.9 \pm 1.6$ & $33.6 \pm 2.8$ \\
& No. 1 & $177.8 \pm 3.4$ & $94.6 \pm 2.4$ \\
& No. 2 & $156.6 \pm 5.0$ & $57.4 \pm 8.9$ \\
& No. 3 & $163.8 \pm 2.7$ & $62.3 \pm 3.5$ \\
& No. 4 & $137.4 \pm 3.3$ & $99.5 \pm 8.2$ \\
& No. 5 & $77.3 \pm 3.1$ & $42.5 \pm 1.0$ \\
\hline
\end{tabular}

A value is calculated to reflect absorption wavelength. The index of $\mathrm{Hb}(\mathrm{IHb})$ changes in the range from 0 to 200 , the index of $\mathrm{SO}_{2}\left(\mathrm{ISO}_{2}\right)$ from 0 to 100 .

(The value correlating to $\mathrm{PO}_{2}$ in the arterial blood is approximately equal to $\mathrm{ISO}_{2} \times 1$ 1.333.) A positive correlation between $\mathrm{IHb}$ and the actual haemoglobin concentration was experimentally confirmed, with a positive correlation between $\mathrm{ISO}_{2}$ and actual $\mathrm{SO}_{2}$ in blood demonstrated.

\section{Results}

Determinations were made every one-half second, and the mean value for 10 seconds was calculated (Table). In the open decompressed state, $\mathrm{SO}_{2}$ values were $40,44,37,34$ and 28 from animals No. 1-No. 5, respectively and haemoglobin concentrations were 178, 156, 164, 137 and 77, respectively. After external moderate dural pressure was applied, $\mathrm{SO}_{2}$ values were 45, 28, 34, 60 and 34, respectively. However, the haemoglobin concentration was 95, 39, 62, 120 and 44 , respectively. That is, $\mathrm{SO}_{2}$ values showed no remarkable change, but haemoglobin concentration values had a tendency to decrease after external dural pressure was applied. (Fig. 2, Fig. 3).

\section{Microscopic observations}

At 3 days, 1 animal was sacrificed and the dural sac was investigated at the pressure site. In cross section, neuronal and vascular structures had become necrotic. The resulting haemorrhagic effect occurred in the grey matter distal from the pressure point and was accompanied by enlargement of the myelin sheath in the white matter.

At 7 days, subdural granulation tissue was noted, the grey matter had necrosed, and many foam cells were seen. In the peripheral nerve cells, chromatolysis and atrophy as degenerative changes were noted.

\section{Discussion}

Measurements of circulation in the spinal cord has been studied by $\mathrm{PO}_{2}$ determination, autoradiography, microangiography, radioisotope, hydrogen 


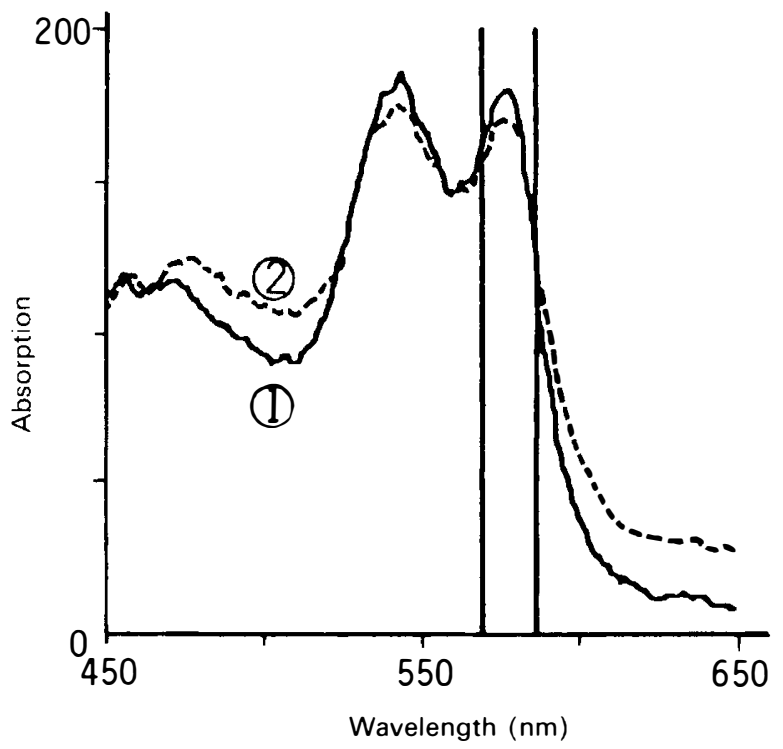

Figure 2 No. 3; Comparison of reflectance spectra pattern (1) in the open decompressed state, (2) after external dural pressure was applied.

clearance method and heat clearance, etc. Among these techniques, all of which have their own characteristics, the hydrogen clearance technique and the heat clearance technique have recently been highlighted. However, determination of blood flow changes in the inner tissue is difficult with both of these techniques because differentation of the white matter of the spinal cord from the grey matter is impossible. However, the tissue reflectance spectrum technique is characterised by its ability to determine minute changes in blood flow and the degree of oxidation and reduction. Therefore, analysis with this technique helps to clarify spinal haemodynamics.

Wagner et al., (1971) reported that the blood vessels in the grey matter are more susceptible to damage from changes in spinal blood flow than are those in the white matter, and that increasing damage results in a reduction of blood flow. Senter and Venes (1979) found that the blood flow begins to decrease about 1 hour after injury because ischaemia is caused by injury-induced oedema, metabolic function and autoregulation.

Suzuki (1985) experimented by compressing the rabbit spinal cord, and found that the blood flow was increased for 1 hour, but decreased 6 hours after compression.

According to Ducker et al., (1981), and Wagner et al., (1971) circulatory disturbances, ischaemia and hypoxia occurred as a result of rupture of the vascular system accompanying injury, causing the degeneration of neurons.

In the present study, laminectomy was undertaken in dogs under general anaesthesia, and the tissue spectrum was determined without compression and then with compression gradually added extradurally. The 5 dogs showed changes in $\mathrm{SO}_{2}$ from 28 to 44 before compression, and 28 to 45 after compression, however, the $\mathrm{Hb}$ concentration index was from 77 to 178 before compression, and from 44 to 120 after compression. 

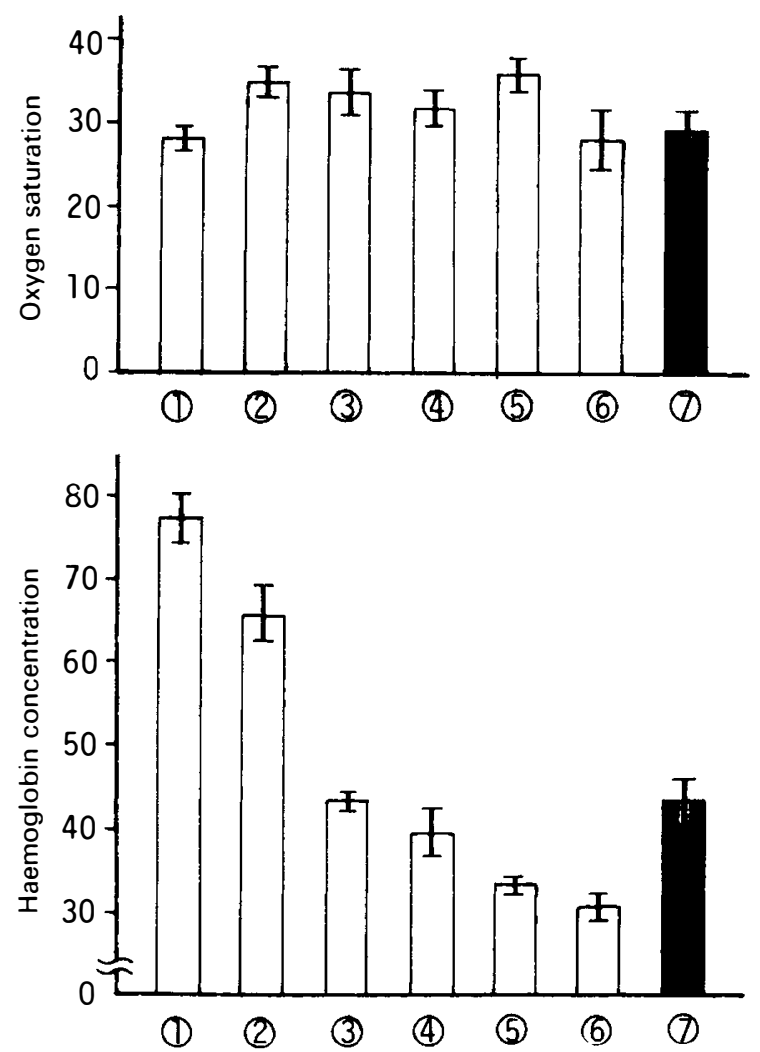

Figure 3 No. 5; Oxygen saturation and haemoglobin concentration (1) in the open decompressed state, (2) after an external 200 gm dural pressure was applied, (3) after an external $600 \mathrm{gm}$ dural pressure was applied, (4) 15 minutes after pressure was applied, (5) 30 minutes after pressure was applied, (6) 1 week after pressure was applied; proximal from pressure level, (7) 1 week after pressure was applied; distal from pressure level.

In the clinical study, most cases of spinal canal stenosis and spondylolisthesis showed only slight changes in $\mathrm{SO}_{2}$ before and after compression; while they showed low haemoglobin concentration under compression, suggesting ischaemia.

Thus in both experimental and clinical studies, the haemoglobin concentration decreased at the peripheral site under compression, whereas $\mathrm{SO}_{2}$ showed no marked changes regardless of compression.

Even if $\mathrm{SO}_{2}$, which indicates the correlation between oxyHb and desoxyHb, does not change, local oxygen consumption varies with haemoglobin level. The phenomenon that $\mathrm{SO}_{2}$ was approximately constant before and after compression raises the following points for further study: why are the oxyHb and desoxyHb levels, being maintained approximately constant, because of general anaesthesia or other factors?

\section{Conclusions}

Haemoglobin concentration and oxygen saturation were determined in the dura 
mater in the open decompressed state and after external dural pressure was applied in dogs using a tissue spectrometer.

It has been confirmed that oxygen saturation was not changed in either state, however, haemoglobin concentration was decreased when external pressure was applied.

\section{References}

Bedbrook GM 1966 Pathological Principles in the Management of Spinal Cord Trauma. Paraplegia 4:43-56.

Chance B 1951 Double beam spectrophotometry. Review of Sicentific Instruments 22:619.

DoHRMANN GJ, et al. 1972 Blood flow patterns in the intrinsic vessels of the spinal cord following contusion: an experimental study. Transactions of the American Neurological Association 97:189-192.

DuCker TB, KINDT GW, Kempe LG 1981 Pathological findings in acute experimental spinal cord trauma. Fournal of Neurosurgery 35:700-708.

HAYASHI N 1979 In vivo studies on the haemodynamics and oxygen utilization in the livers of patients with chronic liver disease-Analysis by reflectance spectrophotometry. Fournal of fapanese Society of Internal Medicine 68:1154-1561.

Hiramoto I Kanda M 1985 Tissue Spectrum Analyzer for Medical Use (Japanese). The Laser Society of Fapan 13:165-170.

MAEDA M 1984 The Application of Reflectance Spectrophotometry to the Behavior of Cutaneous Microcirculation. Skin Research 26:66-73.

Nishijima Y et al. 1982 Studies of Blood Flow through the Spinal Cord of Rabbit Using the $\mathrm{H}_{2}$ Clearance Method. Fournal of the Fapanese Orthopaedic Association 56:213-219.

PanajBi MM 1987 Experimental Spinal Cord Trauma: a Biomechanical Viewpoint. Paraplegia 25:217-220.

Rivin AS, TATOR CH 1978 Regional spinal cord blood flow in rats after severe cord trauma. fournal of Neurosurgery 49:844-853.

SATO N et al. 1979 Measurement of hemoperfusion and oxygen sufficiency in gastric mucosa in vivo. Gastroenterology 76:814-819.

SENTER HJ, VENES JL 1979 Loss of autoregulation and posttraumatic ischemia following experimental spinal cord trauma. Fournal of Neurosurgery 50:198-206.

SUZUKI U 1985 Changes of Microcirculation Dynamics in the Formation of Edema Following Spinal Compression Injury in Rabbits. Fournal of the Fapanese Orthopaedic Association 59:707718.

WAGNER Jr. FG, DOHRMANN G, BUCY PC 1971 Histopathology of transitory traumatic paraplegia in the monkey. Fournal of Neurosurgery 35:272-276.

YAMAGISHI M 1987 The blood flow in normal and injured spinal cord-Experimental studies by the heat clearance method. Fournal of the fapanese Orthopaedic Association 61:1293-1304. 